\title{
EFEITO DE ARILO NA GERMINAÇÃO DE SEMENTES DE Passiflora alata CURTIS EM DIFERENTES SUBSTRATOS E SUBMETIDAS A TRATAMENTOS COM GIBERELINA ${ }^{1}$
}

\author{
GISELA FERREIRA², ANÍSIO DE OLIVEIRA ${ }^{3}$, JOÃO DOMINGOS RODRIGUES ${ }^{4}$, GLÁUCIA BRAVO DIAS ${ }^{5}$, \\ ALESSANDRA MARIA DETONI ${ }^{6}$, SAIONARA MARIA TESSER ${ }^{7}$, ANDRÉA MARIA ANTUNES ${ }^{8}$
}

\begin{abstract}
RESUMO - O objetivo deste trabalho foi avaliar a germinação de sementes de Passiflora alata Curtis sob o efeito da presença de arilo em diferentes substratos de papel e submetidas a pré-tratamentos germinativos. Foram realizados dois experimentos (com e sem remoção de arilo), com delineamento experimental inteiramente casualizado, com 8 tratamentos e 5 repetições de 25 sementes, em esquema fatorial 2x4 (substratos x tratamentos prégerminativos) para cada experimento. Os tratamentos foram constituídos pelos substratos (sobre papel em gerbox e entre papel em rolo) e tratamentos pré-germinativos (sementes embebidas em $\mathrm{GA}_{3}$ e água, papel de germinação umedecido com $\mathrm{GA}_{3}$ e água). Pode-se verificar que a germinação das sementes sem arilo foi maior, em substratos sobre papel ou entre papel, umedecidos com $\mathrm{GA}_{3}$.
\end{abstract}

Termos para indexação: propagação, reguladores vegetais, giberelina, Passiflora sp.

\section{EFFECT OF ARIL IN Passiflora alata SEED GERMINATION IN DIFFERENTS SUBSTRATES AND SUBMITED TO PREVIOUS GERMINATIVE TREATMENTS WITH GIBBERELLIN}

\begin{abstract}
The objective of this work was to evaluate the germination seeds of Passiflora alata submitted to effect of aril presence in different substrates and previous germinated treatments. The work was conduced with two experiments (with and without aril) in a completely randomized design with 8 treatments, 5 replicates per treatment and 25 seeds, in a factorial scheme $2 \mathrm{X} 4$ (substrates x previous germinated treatments) for each experiment. The treatments were composed with the substrates gerbox and roll of paper, and the following previous germinated treatments: seeds soaked in GA3 and in water, paper infusion in GA3 and in water. The analysis of the data showed that the germination has improved when extracting the aril and administrating GA3 infusion in gerbox or roll of paper.
\end{abstract}

Index terms: propagation, plant growth regulator, gibberellins, Passiflora sp.

\section{INTRODUÇÃo}

Com o aumento das áreas de produção de maracujazeiro-doce (Passiflora alata Curtis), exigem-se informações constantes sobre técnicas de propagação, principalmente porque parte das mudas é produzida a partir de sementes e existem relatos de que não apresentam germinação satisfatória (Osipi, 2000).

De acordo com Akamine et al. (1972), as sementes de Passiflora edulis Sims. e P. edulis f. flavicarpa Deg. não requerem limpeza, secagem ou armazenamento antes da semeadura. Porém, os autores afirmaram que a remoção da polpa e a lavagem das sementes aceleram a germinação. São José \& Nakagawa (1988) verificaram que a presença de arilo diminuiu a percentagem de germinação em sementes recém-extraídas de $P$. edulis f. flavicarpa Deg. Deste modo, existem diversos trabalhos com métodos de extração de arilo, como os de Carvalho (1974), Ruggiero \& Correa (1978), Silva (1988), Melo (1996).

Segundo Coneglian et al. (2000), sementes de P. alata submetidas a métodos de extração de arilo e/ou envoltórios, com préembebição em $300 \mathrm{mg} \mathrm{L}^{-1}$ de ácido giberélico e semeadas em papel umedecido com $300 \mathrm{mg} \mathrm{L}^{-1}$ de ácido giberélico, apresentaram maior percentagem de germinação e índice de velocidade de germinação.

Em experimento com a imersão de sementes de Passifloráceas em $\mathrm{GA}_{3}$, citocinina e etileno, Ferreira (1998) verificou, através de análise de componentes principais e de agrupamento, que $\mathrm{GA}_{3}$ e citocinina, isolados ou em mistura, promoveram maior incremento no processo germinativo de P.alata, obtendo-se $85 \%$ de germinação com $100 \mathrm{mg} \mathrm{L}^{-}$ ${ }^{1}$ de $\mathrm{GA}_{3}$.

Melo et al. (2000) obtiveram resultados efetivos na superação da dormência e emergência das plântulas de $P$. nítida através da imersão em solução de 1.500 e $2.000 \mathrm{mg} \mathrm{L}^{-1}$ de $\mathrm{GA}_{3}$. Ferreira et al. (2001b) observaram que sementes de maracujá-doce (Passiflora alata) não tiveram a percentagem de germinação alterada em função do tempo de embebição em $\mathrm{GA}_{3}$, no entanto $500 \mathrm{mg} \mathrm{L}^{-1}$ promoveu a maior percentagem de germinação.

Para a produção de mudas de maracujazeiro-doce (Passiflora alata), Ferreira et al. (2001a) verificaram que, a partir de $100 \mathrm{mg} \mathrm{L}^{-1} \mathrm{de}$ ácido giberélico, se observou aumento na germinação e maior desenvolvimento inicial de mudas em sacos de polietileno preto com substrato comercial.

Pereira \& Andrade (1994) recomendaram utilizar como substrato para semeadura de Passiflora edulis Sims. rolo de papel toalha ou a vermiculita em gerbox, por proporcionarem germinação de $42,3 \%$ e $48,6 \%$, respectivamente, o que diferiu significativamente do tratamento sobre papel de filtro, que apresentou $29,1 \%$ de germinação.

Este trabalho teve por objetivo avaliar a germinação de sementes de Passiflora alata Curtis, sob efeito da presença de arilo, em diferentes substratos de papel e submetidas a pré-tratamentos germinativos.

\section{MATERIAL E MÉTODOS}

O trabalho foi desenvolvido na Universidade Estadual do Oeste do Paraná - UNIOESTE, com sementes extraídas de frutos de maracujazeiro-doce (Passiflora alata Curtis) produzidos no pomar didático, em Pato Bragado - PR.

Foram realizados dois experimentos. No experimento 1, o arilo foi mantido (instalação após abertura do fruto) e, no experimento 2, o arilo foi extraído com uso de liquidificador. As sementes foram lavadas, colocadas à sombra para secar (4 dias) e armazenadas em geladeira (5$10^{\circ} \mathrm{C}$ ), por 3 dias. Antes da semeadura, receberam tratamento antifúngico com Rodhiauran (0,2\%). O delineamento experimental utilizado para ambos os experimentos foi o inteiramente casualisado, com 8 tratamentos e 5 repetições de 25 sementes, em esquema fatorial $2 \times 4$ (substratos $\mathrm{x}$ tratamentos pré-germinativos). Os substratos

\footnotetext{
${ }^{1}$ (Trabalho 007/2005). Recebido: 12/01/2005. Aceito para publicação: 04/08/2005.

${ }^{2}$ Profa. Dra., IB/UNESP- Botucatu, SP, Cx. Postal 510, CEP 18618-000,. Tel (14) 3811-6053. E-mail: gisela@ibb.unesp.br.

${ }^{3}$ Pós-graduando do Programa de Agronomia, UNIOESTE, Mal. C. Rondon-PR

${ }^{4}$ Prof. Dr., IB/UNESP- Botucatu, SP, Cx. Postal 510, CEP 18618-000, Tel (14) 3811-6053. E-mail:mingo@ibb.unesp.br.

$5,6,7$ Acadêmicas do Curso de Agronomia da UNIOESTE, Mal. C. Rondon-PR.

${ }^{8}$ Pós-Graduanda do Programa de Ciências Biológicas (Botânica), Cx. Postal 510, CEP 18618-000, IB/UNESP- Botucatu-SP. Tel (14) 3811-6053. E-mail: andreamantunes@yahoo.com.br.
} 
TABELA 1 - Dados médios de percentagem total de germinação (G), percentagem de sementes que só emitiram raiz primária (SERP) e percentagem de plântulas normais (PN) de sementes de Passiflora alata Curtis, com arilo, em diferentes substratos e submetidas a tratamentos pré-germinativos. Marechal Cândido Rondon - PR, 2001.

\begin{tabular}{|c|c|c|c|c|c|c|}
\hline \multirow[b]{2}{*}{ Pré -tratamentos } & \multicolumn{2}{|c|}{$\mathrm{G}(\%)$} & \multicolumn{2}{|c|}{ SERP $(\%)$} & \multicolumn{2}{|c|}{$\mathrm{PN}(\%)$} \\
\hline & Sobre papel & Rolo de papel & Sobre papel & Rolo de papel & Sobre papel & Rolo de papel \\
\hline Sementes embebidas em GA 3 & $8,0 \mathrm{Ab}^{1}$ & $1,6 \mathrm{Ac}$ & $0,0 \mathrm{Ab}$ & $0,8 \mathrm{Ab}$ & $0,8 \mathrm{Aa}$ & $0,8 \mathrm{Ac}$ \\
\hline Substrato irrigado com GA 3 & $9,6 \mathrm{Ba}$ & $59,2 \mathrm{Aa}$ & $7,2 \mathrm{Ba}$ & $27,2 \mathrm{Aa}$ & $1,6 \mathrm{Ba}$ & $32,0 \mathrm{Aa}$ \\
\hline Substrato irrigado com água & $0,8 \mathrm{Bb}$ & $7,2 \mathrm{Ab}$ & $0,8 \mathrm{Ab}$ & $0,8 \mathrm{Ab}$ & $0,0 \mathrm{Ba}$ & $6,4 \mathrm{Ab}$ \\
\hline Sementes embe bidas em água & $0,0 \mathrm{Bb}$ & $8,0 \mathrm{Ab}$ & $0,0 \mathrm{Ab}$ & $0,0 \mathrm{Ab}$ & $0,0 \mathrm{Ba}$ & $8,0 \mathrm{Ab}$ \\
\hline F Substratos $(\mathrm{S})$ & \multicolumn{2}{|c|}{$110,59 * *$} & \multicolumn{2}{|c|}{$10,52 * *$} & \multicolumn{2}{|c|}{$75,73 * *$} \\
\hline F Pré -tratamentos (PT) & \multicolumn{2}{|c|}{$91,92 * *$} & \multicolumn{2}{|c|}{$57,95 * *$} & \multicolumn{2}{|c|}{$19,21 * *$} \\
\hline F S x PT & \multicolumn{2}{|c|}{$18,91 * *$} & \multicolumn{2}{|c|}{$7,84^{* *}$} & \multicolumn{2}{|c|}{$13,83 * *$} \\
\hline $\mathrm{CV}(\%)$ & \multicolumn{2}{|c|}{25,47} & \multicolumn{2}{|c|}{39,67} & \multicolumn{2}{|c|}{38,41} \\
\hline
\end{tabular}

${ }^{1}$ Médias seguidas de mesma letra maiúscula na linha e minúscula na coluna, dentro de cada fator, não diferem entre si, pelo teste de Tukey, a $1 \%$ de probabilidade.

empregados foram sobre o papel, em gerbox (SP) e entre papel, em rolo (SP), e os tratamentos pré-germinativos foram constituídos de sementes embebidas em água e em solução de $\mathrm{GA}_{3}$ e papel umedecido com solução de $\mathrm{GA}_{3} \mathrm{e}$ água.

Para as sementes embebidas, empregaram-se 5 horas de imersão em solução com $100 \mathrm{mg} \mathrm{L}^{-1}$ de $\mathrm{GA}_{3}$ (i.a.) ou água destilada, sob aeração constante (Ferreira, 1998). A quantidade de solução de $\mathrm{GA}_{3}\left(100 \mathrm{mg} \mathrm{L}^{-1}\right.$ de i.a.) e de água destilada para umedecimento do substrato foi calculada multiplicando-se o peso do papel pelo fator 2,5 (Brasil, 1992). O produto comercial de $\mathrm{GA}_{3}$ usado foi o Pro-Gibb. O teste de germinação foi realizado em câmara, no escuro e com temperaturas alternadas de $20^{\circ} \mathrm{C}$ por 16 horas e $30^{\circ} \mathrm{C}$ por 8 horas.

As avaliações foram realizadas diariamente após a semeadura e a cada dois dias após o início da germinação visível, quantificandose a percentagem total de germinação $(\mathrm{G})$, de plântulas normais $(\mathrm{PN})$ e anormais (PA), sementes dormentes (SD) e mortas (SM), de acordo com Brasil (1992), e o índice de velocidade de germinação (IVG) conforme Silva \& Nakagawa (1995). Foram consideradas sementes germinadas aquelas que apresentavam emissão de radícula até o final do experimento; portanto, independentemente de originarem plântulas normais ou anormais, a semente foi considerada germinada (Bewley $\&$ Black, 1994). No final do experimento, avaliou-se a percentagem de sementes com emissão de raiz primária (\%SERP), tendo em vista que, mesmo após 28 dias da semeadura, havia germinação. Para a avaliação das sementes mortas, realizou-se o teste de tetrazólio. As sementes embebidas em água por 24 horas foram cortadas longitudinalmente, colocadas para colorir em solução de tetrazólio a $0,075 \%$ por três horas e realizadas as leituras de acordo com a escala diagramática de Malavasi et al. (2001).

Os dados de percentagem foram transformados em $\sqrt{x \quad 0,50} \mathrm{e}$ os resultados submetidos à análise de variância, sendo as médias comparadas pelo teste de Tukey, a $1 \%$ e $5 \%$ de probabilidade.

\section{RESULTADOS E DISCUSSÃO}

Observa-se no experimento 1 que, sem a extração de arilo, ocorreram interações significativas entre substratos e pré-tratamentos para os parâmetros G, SERP, PN (Tabela 1), SM e IVG (Tabela 2), cujos maiores valores, exceto para SM, foram obtidos com o substrato umedecido com $\mathrm{GA}_{3}$ no rolo de papel. Para SD, não ocorreram interações significativas (Tabela 3), portanto os resultados estão apresentados separadamente, e os menores valores foram obtidos com emprego do rolo de papel e substrato umedecido com GA. Neste experimento, os tratamentos não provocaram diferenças significativas na percentagem de plântulas anormais, o que demonstra não ter havido efeito negativo dos tratamentos (Tabela 3).

No experimento 2, verifica-se que as maiores percentagens de germinação foram obtidas com substrato irrigado com $\mathrm{GA}_{3}$, tanto sobre o papel como no rolo de papel, que não diferiram significativamente entre si (Tabela 4). Tais resultados são confirmados
TABELA 2 - Dados médios de percentagem de sementes mortas (SM) e índice de velocidade de germinação de sementes de Passiflora alata Curtis, com arilo, em diferentes substratos e submetidas a tratamentos pré-germinativos. Marechal Cândido Rondon - PR, 2001.

\begin{tabular}{|c|c|c|c|c|}
\hline \multirow[t]{2}{*}{ Pré -tratamentos } & \multicolumn{2}{|c|}{ SM (\%) } & \multicolumn{2}{|c|}{ IVG } \\
\hline & $\begin{array}{l}\text { Sobre } \\
\text { papel }\end{array}$ & $\begin{array}{l}\text { Rolo } \\
\text { papel }\end{array}$ & $\begin{array}{l}\text { Sobre } \\
\text { pape l }\end{array}$ & $\begin{array}{l}\text { Rolo } \\
\text { papel }\end{array}$ \\
\hline Sem. emb. GA 3 & $12,6 \mathrm{Ab}^{1}$ & $12,4 \mathrm{Aab}$ & $0,0035 \mathrm{Aa}$ & $0,064 \mathrm{Ab}$ \\
\hline Subst. umed. GA 3 & $38,2 \mathrm{Aa}$ & $4,0 \mathrm{Bb}$ & $0,879 \mathrm{Ba}$ & $6,093 \mathrm{Aa}$ \\
\hline Subst. umed. água & $13,8 \mathrm{Ab}$ & $15,8 \mathrm{Aa}$ & $0,, 83 \mathrm{Aa}$ & $0,232 \mathrm{Ab}$ \\
\hline Sem. emb. água & $7,2 \mathrm{Ab}$ & $6,4 \mathrm{Aab}$ & $0,000 \mathrm{Aa}$ & $0,208 \mathrm{Ab}$ \\
\hline F Substratos (S) & \multicolumn{2}{|c|}{$9,60 * *$} & \multicolumn{2}{|c|}{$78,73 * *$} \\
\hline F Pré - trat (PT) & \multicolumn{2}{|c|}{$3,54^{*}$} & \multicolumn{2}{|c|}{$136,33^{* *}$} \\
\hline $\mathrm{F} \mathrm{S} \times \mathrm{PT}$ & \multicolumn{2}{|c|}{$9,18^{* *}$} & \multicolumn{2}{|c|}{$50,64 * *$} \\
\hline CV $(\%)$ & \multicolumn{2}{|c|}{34,22} & \multicolumn{2}{|c|}{1407} \\
\hline
\end{tabular}

${ }^{1}$ Médias seguidas de mesma letra maiúscula na linha e minúscula na coluna, dentro de cada fator, não diferem entre si, pelo teste de Tukey, a $1 \%$ e $5 \%$ de probabilidade.

TABELA3 - Dados médios de percentagem de plântulas anormais (PA), percentagem de sementes dormentes (SD) de Passiflora alata Curtis, com arilo, em diferentes substratos e submetidas a tratamentos pré-germinativos. Marechal Cândido Rondon-PR, 2001.

\begin{tabular}{lcc}
\hline Substratos & PA $(\%)$ & SD $(\%)$ \\
\hline Sobre papel & $0,778 \mathrm{a}$ & $78,75 \mathrm{a}^{1}$ \\
Rolo de papel & $0,707 \mathrm{a}$ & $70,15 \mathrm{~b}$ \\
$\mathrm{~F}$ & $1 \mathrm{~ns}$ & $10,15^{* *}$ \\
$\mathrm{CV}(\%)$ & 30,11 & 11,21 \\
\hline Pré-tratamentos & $\% \mathrm{PA}$ & $\% \mathrm{SD}$ \\
\hline Sementes embebidas em $\mathrm{GA}_{3}$ & $0,707 \mathrm{a}$ & $85,10 \mathrm{a}$ \\
Substrato umedecido com $\mathrm{GA}_{3}$ & $0,848 \mathrm{a}$ & $43,50 \mathrm{~b}$ \\
Substrato umedecido com água & $0,707 \mathrm{a}$ & $80,40 \mathrm{a}$ \\
Sementes embebidas em água & $0,707 \mathrm{a}$ & $88,40 \mathrm{a}$ \\
$\mathrm{F}$ & $1 \mathrm{~ns}$ & $62,41^{* *}$ \\
CV $(\%)$ & 30,11 & 11,21 \\
\hline
\end{tabular}

${ }^{1}$ Médias seguidas de mesma letra minúscula na coluna não diferem entre si, pelo teste de Tukey, a $1 \%$ de probabilidade.

pela percentagem de plântulas normais (Tabela 4) e pelo IVG que, embora sem interações significativas (Tabela 5), apresentaram os maiores valores em substrato sobre papel e substrato umedecido com $\mathrm{GA}_{3}$. Além disso, verifica-se baixa percentagem de sementes com início de emissão de raiz (SERP) com esses tratamentos (Tabela 4), o que se justifica pela elevada germinação com emprego de substrato sobre papel umedecido com $\mathrm{GA}_{3}(83,2 \%)$ e rolo de papel $(84,0 \%)$ logo no início das avaliações e, conseqüentemente, maiores IVG e menores SD e SM (Tabela 6).

Considerando-se os dois experimentos, observa-se, de modo 
TABELA 4 - Dados médios de percentagem total de germinação $(\mathrm{G})$, percentagem de sementes que só emitiram raiz primária (SERP) e percentagem de plântulas normais (PN) de Passiflora alata Curtis, sem arilo, em diferentes substratos e submetidas a tratamentos pré-germinativos. Marechal Cândido Rondon - PR, 2001.

\begin{tabular}{|c|c|c|c|c|c|c|}
\hline \multirow[b]{2}{*}{ Pré -tratamentos } & \multicolumn{2}{|c|}{$\mathrm{G}(\%)$} & \multicolumn{2}{|l|}{ SERP (\%) } & \multicolumn{2}{|c|}{$\mathrm{PN}(\%)$} \\
\hline & Sobre papel & Rolo de papel & Sobre papel & Rolo de papel & Sobre papel & Rolo de papel \\
\hline Sementes embebidas em GA 3 & $64,0 \mathrm{Aa}^{1}$ & $34,4 \mathrm{Bb}$ & $0,8 \mathrm{Bb}$ & $4,8 \mathrm{Aa}$ & $58,4 \mathrm{Ab}$ & $29,6 \mathrm{Bb}$ \\
\hline Substrato umedecido com GA 3 & $83,2 \mathrm{Aa}$ & $84,0 \mathrm{Aa}$ & $0,8 \mathrm{Ab}$ & $0,8 \mathrm{Aab}$ & $80,0 \mathrm{Aa}$ & $83,2 \mathrm{Aa}$ \\
\hline Substrato umedecido com água & $29,8 \mathrm{Ab}$ & $32,8 \mathrm{Ab}$ & $10,4 \mathrm{Aa}$ & $5,6 \mathrm{Aa}$ & $17,8 \mathrm{Ac}$ & $27,2 \mathrm{Ab}$ \\
\hline Sementes embebidas em água & $32,8 \mathrm{Ab}$ & $17,4 \mathrm{Bc}$ & $11,2 \mathrm{Aa}$ & $0,0 \mathrm{Bb}$ & $21,6 \mathrm{Ac}$ & $14,4 \mathrm{Ac}$ \\
\hline F Substratos (S) & \multicolumn{2}{|c|}{$18,74 * *$} & \multicolumn{2}{|l|}{$4,41 *$} & \multicolumn{2}{|c|}{$4,37^{*}$} \\
\hline F Pré -tratamentos $(\mathrm{PT})$ & \multicolumn{2}{|c|}{$83,23 * *$} & \multicolumn{2}{|l|}{$7,45^{* *}$} & \multicolumn{2}{|c|}{$92,38 * *$} \\
\hline $\mathrm{F} \mathrm{S} \times \mathrm{PT}$ & \multicolumn{2}{|c|}{$8,48 * *$} & \multicolumn{2}{|l|}{$9,68 * *$} & \multicolumn{2}{|c|}{$8,45^{* *}$} \\
\hline $\mathrm{CV}(\%)$ & \multicolumn{2}{|c|}{10,08} & \multicolumn{2}{|l|}{44,97} & \multicolumn{2}{|c|}{11,72} \\
\hline
\end{tabular}

${ }^{1}$ Médias seguidas de mesma letra maiúscula na linha e minúscula na coluna, dentro de cada fator, não diferem entre si, pelo teste de Tukey, a $1 \%$ e $5 \%$ de probabilidade.

TABELA 5 - Dados médios de percentagem de plântulas anormais (PA), IVG de sementes de Passiflora alata Curtis, sem arilo, em diferentes substratos e submetidas a tratamentos pré-germinativos. Marechal Cândido Rondon $-\mathrm{PR}, 2001$.

\begin{tabular}{lcc}
\hline Substratos & PA $(\%)$ & IVG \\
& & \\
\hline Sobre papel & $1,39 \mathrm{a}^{1}$ & $9,56 \mathrm{a}$ \\
Rolo de papel & $0,71 \mathrm{~b}$ & $7,10 \mathrm{~b}$ \\
$\mathrm{~F}$ & $9,97^{* *}$ & $23,92^{* *}$ \\
$\mathrm{CV}(\%)$ & 65,36 & 12,74 \\
\hline Pré-tratamentos & $\% \mathrm{PA}$ & $\mathrm{IVG}$ \\
\hline Sementes embebidas em $\mathrm{GA}_{3}$ & $1,35 \mathrm{a}$ & $8,65 \mathrm{~b}$ \\
Substrato umedecido com $\mathrm{GA}_{3}$ & $1,07 \mathrm{a}$ & $20,25 \mathrm{a}$ \\
Substrato umedecido com água & $1,07 \mathrm{a}$ & $2,44 \mathrm{c}$ \\
Sementes embebidas em água & $0,71 \mathrm{a}$ & $2,18 \mathrm{c}$ \\
F & $1,48 \mathrm{~ns}$ & $161,71^{* *}$ \\
CV (\%) & 65,36 & 12,74 \\
\hline
\end{tabular}

${ }^{1}$ Médias seguidas de mesma letra minúscula na coluna não diferem entre si, pelo teste de Tukey, a $1 \%$ de probabilidade.

TABELA 6 - Dados médios de porcentagem de sementes dormentes (SD) e porcentagem de sementes mortas (SM) de sementes de Passiflora alata Dryander, sem arilo, em diferentes substratos e submetidas a tratamentos prégerminativos. Marechal Cândido Rondon - PR, 2001.

\begin{tabular}{lllll}
\hline Pré -tratamentos & \multicolumn{2}{c}{ SD (\%) } & \multicolumn{2}{c}{ SM (\%) } \\
\cline { 2 - 5 } & $\begin{array}{l}\text { Sobre } \\
\text { papel }\end{array}$ & $\begin{array}{l}\text { Rolo } \\
\text { papel }\end{array}$ & $\begin{array}{l}\text { Sobre } \\
\text { papel }\end{array}$ & $\begin{array}{l}\text { Rolo } \\
\text { papel }\end{array}$ \\
\hline Sem. emb. GA 3 & $23,2 \mathrm{Bb}^{1}$ & $54,6 \mathrm{Aa}$ & $8,8 \mathrm{Ab}$ & $11,0 \mathrm{Aa}$ \\
Subst. umed. GA & $11,2 \mathrm{Ab}$ & $12,0 \mathrm{Ab}$ & $4,8 \mathrm{Ab}$ & $4,0 \mathrm{Aa}$ \\
Subst. umed. água & $53,8 \mathrm{Aa}$ & $54,6 \mathrm{Aa}$ & $14,8 \mathrm{Aa}$ & $12,6 \mathrm{Ba}$ \\
Sem. emb. água & $61,2 \mathrm{Aa}$ & $70,6 \mathrm{Aa}$ & $5,2 \mathrm{Aa}$ & $14,2 \mathrm{Ba}$ \\
\hline F Substratos (S) & \multicolumn{2}{c}{$6,53^{*}$} & \multicolumn{2}{c}{$25,15^{* *}$} \\
F Pré - trat (PT) & \multicolumn{2}{c}{$45,90^{* *}$} & \multicolumn{2}{c}{$15,73^{* *}$} \\
F S x PT & \multicolumn{2}{c}{$3,76^{*}$} & \multicolumn{2}{c}{$9,91^{* *}$} \\
CV(\%) & \multicolumn{2}{c}{16,06} & \multicolumn{2}{c}{33,10} \\
\hline
\end{tabular}

${ }^{1}$ Médias seguidas de mesma letra maiúscula na linha e minúscula na coluna, dentro de cada fator, não diferem entre si, pelo teste de Tukey, a 1\% e 5\% de probabilidade.

geral, que a remoção do arilo foi benéfica ao processo germinativo, promovendo os maiores valores, o que confirma a remoção de substâncias inibidoras da germinação juntamente com a extração do arilo, e está de acordo com Akamine et al. (1972), São José \& Nakagawa (1988), Melo (1996), entre outros.

Com a manutenção do arilo, observa-se que o umedecimento do substrato rolo de papel com $\mathrm{GA}_{3}$ foi o tratamento que incrementou significativamente a germinação $(59,2 \%)$ embora este valor seja menor do que os obtidos com $\mathrm{GA}_{3}$ sobre papel $(83,2 \%)$ e no rolo de papel $(84,0 \%)$ em sementes sem arilo, demonstrando o efeito benéfico do $\mathrm{GA}_{3}$. Considerando, portanto, que o $\mathrm{GA}_{3}$ estimula a síntese de enzimas como a alfa amilase e a liberação de energia para a retomada do crescimento do embrião e conseqüente germinação (Salisbury \& Ross, 1992; Taiz \& Zeiger, 2004), o ácido giberélico $\left(\mathrm{GA}_{3}\right)$ pode ser empregado no substrato para estimular a germinação mesmo em sementes com arilo, embora outras concentrações devam ser estudadas para atingir valores de germinação próximos àqueles aqui obtidos com a retirada de arilo e o emprego de $100 \mathrm{mg} \mathrm{L}^{-1} \mathrm{de} \mathrm{GA}_{3}$, conforme verificado em trabalhos de Ferreira (1998), Coneglian et al. (2000), Ferreira et al. (2001b) e Melo et al. (2000).

Observa-se que, como ocorreram interações significativas, deve-se ter critério na escolha do substrato em função do prétratamento empregado, pois enquanto Pereira \& Andrade (1994) obtiveram maior percentagem de germinação em rolo de papel, neste trabalho, verificou-se que ambos os substratos podem ser empregados, desde que retirado o arilo da semente e acrescido de $\mathrm{GA}_{3}$ em umedecimento do papel.

\section{CONCLUSÕES}

A germinação das sementes de Passiflora alata Curtis foi incrementada com a extração do arilo, semeadura sobre papel em gerbox ou entre papel em rolo e com umedecimento do substrato com $\mathrm{GA}_{3}$.

\section{REFERÊNCIAS}

AKAMINE,E.K.; ARAGAKI, M.; BEUMONT, J.H.; BOWERS, F.A.I.; HAMILTON, R.A.; NISHIDA, T.; SHERMAN, G.D.; SHOJI, K.; SOTREY, W.B. Passion fruit culture in Hawaii. Coop. Extension Service Circular, Califórnia, n.345, p.1-35, 1972.

BEWLEY, J.D.; BLACK, M. Seeds: physiology of development and germination. New York: Plenum Press, 1994. 445p.

BRASIL. Ministério da Agricultura e Reforma Agrária. Regras para análise de sementes. Brasília: SNDA/DNDV/CLAV, 1992.365p.

CARVALHO, A. M. Melhoramento cultural do maracujazeiro. In: SIMPÓSIO DA CULTURA DO MARACUJÁ, 1., 1974, Campinas. Anais... Campinas: Sociedade Brasileira de Fruticultura, 1974. p.1-9.

CONEGLIAN, R.C.C.; ROSSETTO, C.A.V.; SHIMIZU, M.K.; VASCONCELLOS, M.A.S. Efeitos de métodos de extração e de ácido giberélico na qualidade de sementes de maracujá-doce (Passiflora alata Dryander). Revista Brasileira de Fruticultura, Jaboticabal, v. 22, n.3, p.463-467, 2000.

FERREIRA, G. Estudo da embebição e do efeito de fitorreguladores na germinação de sementes de Passifloráceas. 1998. 144f. Tese (Doutorado em Horticultura), Faculdade de Ciências Agronômicas, Universidade Estadual Paulista, Botucatu, 1998.

FERREIRA, G.; FOGAÇA, L. A.; BLOEDORN, M. Efeito do ácido 
giberélico $\left(\mathrm{GA}_{3}\right)$ aplicado em sementes de maracujá-doce (Passiflora alata Dryander) para a produção de mudas em diferentes embalagens. Revista Brasileira de Fruticultura, Jaboticabal, v.23, n.1,p.152-155,2001a.

FERREIRA, G.; FOGAÇA, L. A.; MORO, E. Germinação de sementes de Passiflora alata Dryander (maracujá doce) submetidas a diferentes tempos de embebição e concentrações de ácido giberélico. Revista Brasileira de Fruticultura, Jaboticabal, v.23, n.1, p.160-163, 2001b.

MALAVASI, M. M.; FOGAÇA, C. A.; FOGAÇA L. A.; FERREIRA, G. Preparo e coloração de sementes de maracujá-doce (Passiflora alata Dryander) para a avaliação da viabilidade através do teste de tetrazólio. Revista Brasileira de Fruticultura, Jaboticabal, v.23, n.1, p. 126-129, 2001.

MELO, A.L. Efeitos da retirada do arilo e do armazenamento e aspectos morfológicos de sementes do maracujazeiro (Passiflora spp.). 1996. 52f. Dissertação (Mestrado em Produção e Tecnologia de Sementes) - Faculdade de Ciências Agrárias e Veterinárias, Universidade Estadual Paulista, Jaboticabal, 1996.

MELO, A.L.; OLIVEIRA, J.C.; VIEIRA, R.D. Superação de dormência em sementes de Passiflora nitida H.B.K. com hidróxido de cálcio, ácido sulfúrico e ácido giberélico. Revista Brasileira de Fruticultura, Jaboticabal, v. 22, n.2, p.463-467, 2000.

OSIPI, E.A.F. Efeito da temperatura, da maturação do fruto e do armazenamento na qualidade fisiológica de sementes de maracujádoce (Passiflora alata Dryander). 2000. 98f. Tese (Doutorado em
Horticultura) - Faculdade de Ciências Agronômicas, Universidade Estadual Paulista, Botucatu, 2000.

PEREIRA, T.S; ANDRADE, A.C.S. Germinação de Psidium guajava L. e Passiflora edulis Sims - efeito da temperatura, substrato e morfologia do desenvolvimento pós-seminal. Revista Brasileira de Sementes, Brasília, v.16 n.l, p.58-62, 1994.

RUGGIERO, C.; CORREA, L.S. Propagação do maracujazeiro. In: SIMPÓSIO SOBREACULTURADO MARACUJAZEIRO, 2., 1978, Jaboticabal. Anais... Jaboticabal: Faculdade de Ciências Agrárias e Veterinárias, Universidade Estadual Paulista, 1978. p.24-8.

SALISBURY, F.B.; ROSS, C.W. Plant physiology. $4^{\text {th }}$ ed. Califórnia: Wadsworth, 1992.682p.

SÃO JOSÉ, A.R.; NAKAGAWA, J. Influência do método de extração na qualidade fisiológica de sementes de maracujazeiro-amarelo. In: CONGRESSO BRASILEIRO DE FRUTICULTURA, 9., 1987, Campinas. Anais... Campinas: Sociedade Brasileira de Fruticultura, 1988. p.619-623.

SILVA, R.F. Extração de sementes de frutos carnosos. In: CARVALHO,N.M.; NAKAGAWA, J. Sementes: ciência, tecnologia e produção. Campinas: Fundação Cargill,1988. p. 328-49.

SILVA, J. B. C.; NAKAGAWA, J. Estudo de fórmulas para cálculos da velocidade de germinação. Informativo ABRATES, Londrina, v 5, n.1, p.62-73, 1995.

TAIZ, L.; ZEIGER, E. Fisiologia vegetal. 3.ed. Porto Alegre: Artmed, 2004.719p. 